\title{
Configuración sintáctica de los enunciados exclamativos
}

\author{
Pedro Carbonero Cano
}

\section{Introducción}

En este trabajo pretendemos describir y explicar las configuraciones sintácticas que presenta en español una de las modalidades oracionales menos estudiadas y de las que se conoce poco más que las generalidades de las gramáticas o manuales: la exclamativa.

Existen, no obstante, algunas investigaciones en diferentes lenguas como, por ejemplo, las de J. C. Milner ${ }^{1}$, A. Culioli ${ }^{2}$ y J. Gérard ${ }^{3}$ sobre el francés, D. Elliot ${ }^{4}$ sobre el inglés, A. Radford ${ }^{5}$ sobre el italiano, D. Zaefferer ${ }^{6}$ sobre el alemán, así como I. Bosque $^{7}$ sobre algunas estructuras del español.

${ }^{1}$ J.C. Milner: De la syntaxe à l' interprétation. Paris, Seuil, 1974; «Les exclamatives et le complementizer», en C. Rohrer y N. Ruwet (eds.): Actes du Colloque Franco-Allemand de Grammaire Transformationnelle. Tübingen, Max Niemeyer, 1 (1974), 78-121; «De la interpretación exclamativa como valor semántico residual», en N. Chomsky y otros: La teoría estándar extendida. Madrid, Cátedra, 1979, 117-130.

${ }^{2}$ A. Culioli: «A propos des énoncés exclamatifs», Langue Française, 22 (1974), 6-15.

${ }^{3}$ J. Gérard: L'exclamation en Français. Tübingen, Max Niemeyer, 1980.

${ }^{4}$ D. Elliot: «Toward a grammar of exclamations», Foundations of Language, 11 (1974), 231-246.

5 A. Radford: «The syntax of verbal wh-exclamatives in Italian», en N. Vincent y M. Harris (eds.): Studies in Romance Verb. London, Croom Helm, 1982, 185-204.

${ }^{6}$ D. Zaefferer: «The semantics of sentence mood: investigating german exclamatories», En Bäuerle, Rainer y otros (eds.): Meaning, Use and Interpretation on Language. Berlin, W. de Gruyter, 1983.

${ }^{7}$ I. Bosque: «Sobre la sintaxis de las oraciones exclamativas», Hispanic Linguistics, 1-2 (1984), 283304. 
Haciendo una revisión de las gramáticas y otros tratados de nuestra lengua, observamos que no siempre el exclamativo queda claramente considerado como un tipo de enunciados que posea sus propios rasgos de estructuración sintáctica. A veces se ven caracterizados sólo por poseer determinados aspectos entonativos, aplicables a las distintas clases de modalidad (declarativa, optativa, imperativa...).

En nuestra investigación partimos de la hipótesis de que los enunciados exclamativos poseen una «forma de expresión» gramatical diferenciada, que va asociada a una determinada «forma de contenido» modal ${ }^{8}$, es decir, una interpretación semántica peculiar, que consiste fundamentalmente en la manifestación de un «juicio afectivo». ${ }^{9}$

Es suficiente, para adherirse inicialmente a esta hipótesis, con una comprobación. Tomemos una serie de oraciones de las que oralmente posean entonación exclamativa o por escrito aparezcan con los correspondientes signos de admiración:

/1/ a. ¡Lo aprecia como a un hermano!

b. ¡Ojalá vuelva pronto!

c. ¡Calla, hombre!

/2/ a. ¡Qué guapa está!

b. ¡Cuánto me duele!

c. ¡Se ha puesto más huraño!

d. ¡Los discos que tiene!

Hay un grupo de ellas que, si son despojadas de su marca entonativa o gráfica, perderán desde luego la fuerza afectiva que tenían y se quedarán en simples oraciones con su correspondiente valor modal (declarativo en $/ 1 \mathrm{a} /$, optativo en $/ 1 \mathrm{~b} /$, yusivo en $/ 1 \mathrm{c} /$, etc.). Pero habrá otras, como las del grupo /2/, las cuales, desprovistas de su marca entonativa o gráfica, manifestarán una estructura gramatical especial que -al ser enunciadas como sintácticamente independientes- las seguirá caracterizando como exclamativas.

Pornecesidades de delimitación metodológica estudiaremos sólo lo que concierne a la estructuración sintáctica de este tipo de enunciados y no entramos en el análisis fonético de los mismos, lo cual decididamente es un aspecto relevante pero que sería motivo de una investigación específica muy distinta.

Por otra parte, al ser nuestro propósito conocer los esquemas de estructuración sintáctica, son objeto de esta investigación aquellos enunciados donde haya una construcción sintagmática susceptible de ser analizada en componentes, posean o no una estructura predicativa oracional. Por ello, quedan fuera de nuestro alcance las interjecciones (jeh!, iuf!, jbravo!...) o las frases hechas ( jvaya tela!, ;no veas!...) que presenten un fuerte grado de lexicalización.

${ }^{8}$ Cf. J.M. González Calvo: «Hacia una clasificación de la oración simple según el modus», Serta Philologica F. Lázaro Carreter, 1 (1983), 251-262.

9 Utilizamos la denominación de «juicio afectivo» según Ch. Bally: Linguistique Générale et Linguistique Française. Berne, Francke, 1965. 
Ante la diversidad de configuraciones con que podemos encontrarnos, se impone la tarea de llegar a una visión organizada y darles una explicación sintáctica y semántica. Desde un punto de vista formal, hay que diferenciar inicialmente dos grandes grupos, según se construyan, respectivamente, con o sin «partícula exclamativa».

\section{Enunciados con partícula exclamativa}

Las estructuras con partículas, que suelen llamarse convencionalmente «formas $q u$-», poseen una apariencia sintáctica similar a las interrogativas, en concreto las tradicionalmente llamadas interrogativas parciales, como en:

/3/ a. ¡Cómo lo sabes!

b. ¿Cómo lo sabes?

c. ¡Cuánto has estudiado!

d. ¿Cuánto has estudiado?

Obsérvese que en $/ 3 \mathrm{~b} / \mathrm{y} / 3 \mathrm{~d} /$ se expresa una ignorancia del hablante, mientras que $\mathrm{Ba} / \mathrm{y} / \mathrm{c} /$ indican certeza del hablante ante lo expresado. Pero, a pesar de paralelismos como los anteriores, ya algunos autores, especialmente D. Elliot ${ }^{10} \mathrm{e}$ I. Bosque ${ }^{11}$, se han detenido a observar algunas distinciones entre las esctructuras interrogativas y las exclamativas. Hay muchos tipos de enunciados exclamativos -como iremos viendo seguidamente- cuya configuración no es posible en los interrogativos:

$1^{\circ}$ ) Una de las diferencias más palpables es la que se refiere a la partícula que seleccionan algunas categorías gramaticales. Concretamente, en español actual, la forma qué puede acompañar a adjetivos y adverbios en la exclamación y no así en la interrogación. Contrástese:

/4/ a. ¡Qué alto está!

b. ¡Qué temprano ha llegado!

c. *¿Qué alto está?

d. *¿Qué temprano ha llegado?

La estructura interrogativa que correspondería a $/ 4 \mathrm{a} / \mathrm{o} / 4 \mathrm{~b} /$ no sería $/ 4 \mathrm{c} / \mathrm{o} / 4 \mathrm{~d} /$, sino $15 \mathrm{a} /$ y $/ 5 \mathrm{~b} /$, la cual, por su parte, sí puede servir para formar enunciados exclamativos:

15/ a. ¿Cómo está de alto?

b. ¿Cómo ha llegado de temprano?

c. ¡Cómo está de alto!

d. ¡Cómo ha llegado de temprano!

${ }^{10}$ D. Elliot, op. cit.

${ }^{11}$ I. Bosque: «Sobre la interrogación indirecta», Dicenda, 1 (1982), 13-34; «La selección de las palabras interrogativas», Verba, 11 (1984), 245-273. 
$2^{\circ}$ ) Ciertas formas oracionales que son similares para la interrogación y la exclamación se diferencian a instancias de las condiciones pragmáticas del discurso, condiciones que -además de las marcas suprasegmentales evidentes- pueden verse marcadas gramaticalmente. Véase, por ejemplo la pertinencia de la persona verbal en pares como los siguientes:

/6/ a. ¡Cuánto has trabajado hoy!

b. ¿Cuánto has trabajado hoy?

I7/ a. ¡Cuánto lo siento!

b. ¿(Que) cuánto lo siento?

Claramente /6b/ es el correlato interrogativo de /6a/, ya que la formulación de la segunda persona en el verbo admite suponer desconocimiento del hablante acerca de lo interrogado, pues se hace una pregunta con base en el oyente. Pero en $/ 7 \mathrm{~b} /$, con verbo en primera persona, habría una incongruencia pragmática en el hecho de que el hablante planteara a su interlocutor una pregunta con base en el propio hablante. Por tanto $/ 7 \mathrm{~b} /$ resulta una pregunta poco probable, a no ser en un contexto de interrogación retórica o, sobre todo, como un tipo de las que algunos llaman «interrogativas eco» ${ }^{12}$, las cuales se caracterizan por admitir facultativamente una forma que no acentuada precediendo a la partícula interrogativa, ya que se hacen eco de una pregunta previamente formulada por el interlocutor. Así, /7b/ podría ser una réplica a una pregunta previa:

/7/ c. ¿Cuánto lo sientes?

$3^{\text {o) }}$ En muchos contextos discursivos realmente los enunciados exclamativos se caracterizan por la presencia de elementos valorativos (como el que subrayamos en $/ 8 \mathrm{a} /$ ), interjectivos (como en $/ 8 \mathrm{~b} /$ ) o vocativos (como en $/ 8 \mathrm{c} /$ ) que -además de la entonación-favorecen, aunque no obliguen a ello, la interpretación exclamativa, puesto que tales elementos acentúan el carácter afectivo o emotivo de la frase, lo cual es propio del contenido modal de la exclamación:

/8/ a. ¡Cómo picó, inocente!

b. Yo estoy temiendo este año, porque a mí me van a poner... iuy, cómo me van a poner!

c. ¡Ay, Cecilio, qué raro estás hoy!

\subsection{Tipología estructural}

En principio, una forma qu- puede acompañar a cualquiera de las cuatro categorías designativas básicas de la lengua: sustantivo (¡cuánta gente!), adjetivo (¡qué grande!),

${ }^{12}$ Cf. A. Radford: Introducción a la sintaxis transformativa. Barcelona, Teide, 1988, pág. 144. 
verbo (¡cómo lloraba!) o adverbio (¡qué bien!). Pero para conseguir la tipología sintáctica de estos enunciados se hace necesario estudiar las posibilidades y restricciones combinatorias de cada una de las partículas (qué, cómo, cuán, quién... $)^{13}$ con las mencionadas categorías gramaticales.

Como ya apunta D. Elliot ${ }^{14}$ para el inglés, no todas las formas $q u$ - de la lengua son igualmente válidas para contituir enunciados propiamente exclamativos. De nuestra investigación hemos podido obtener que son posibles las formas qué, cuánto, cómo, cuán, quién y dónde, pero no tan claramente lo son otras como cuándo, porqué, para qué o cuál. Observemos construcciones como las siguientes:

19/ a. ¡Qué calor hace!

b. ¡Cuánto has tardado!

c. ¡Cómo se explica!

d. ¡Cuán hermosa es!

e. ¡Quiénes han venido!

f. ¡Dónde viven!

/10/ a. ¡Cuándo acabará todo esto!

b. ¡Porqué se pondría ella así!

c. ¡Para qué dije aquello!.

Hay un rasgo fundamental de contenido modal que diferencia cada uno de los dos grupos anteriores. Los enunciados de /9/-que consideramos propiamente exclamativospueden ser interpretados con el rasgo semántico de «factividad», lo cual implica una certeza del hablante (el hablante no ignora, sino que se admira del calor que hace -en /9a/- o del lugar donde viven -en /9f/-, etc.). Por el contrario, los de /10/ sólo puẹden interpretarse a través del rasgo semántico de «problematicidad» (el hablante ignora, esto es, se pregunta cuándo acabará todo esto o porqué se pondría ella así, etc.). Por tanto, estos últimos, aunque pueden ser expresados con entonación exclamativa o con signos de admiración, son por su contenido modal enunciados interrogativos ${ }^{15}$. Funciona, pues, aquí el rasgo opositivo «factividad»/«problematicidad». que consideramos básico para la distinción semántica entre exclamación e interrogación.

Una prueba de ello se puede encontrar en que las oraciones de /10/ no pierden esencialmente su contenido modal si se incluyen como subordinadas en estructuras como las interrogativas indirectas ${ }^{16}$ :

${ }^{13}$ Las llamamos partículas, a pesar de la escasa precisión de este término, ya que no pertenecen a una sola «parte de la oración» $y$, como se ha visto, pueden presentar un funcionamiento pronominal, adjetivo o adverbial.

${ }^{14}$ D. Elliot, op. cit.

${ }^{15}$ Son las denominadas preguntas exclamativas por S. Femández Ramírez: Gramática Española, 4. El verbo y la oración. Madrid, Arco-Libros, 1986, 485-491.

16 Un asunto que no vamos a estudiar en este trabajo es el de las «exclamativas indirectas», que merecerían un tratamiento detenido. 
/11/a. No sé cuándo acabará todo esto.

b. Me pregunto porqué se pondría así.

Pero si hiciéramos esta misma transformación con las de /9/ se produciría una variación semántica, consistente en la pérdida de la «certeza» que inicialmente contenían.

De entre las formas qu- que antes citamos como posibles, debemos señalar que quién y dónde no son muy habituales para la exclamación y que, por su parte, la forma cuán queda para usos arcaicos o literarios. Esto quiere decir que en el español actual el mayor rendimiento en las exclamativas qu-se obtiene con las formas qué, cuánto y cómo. Pero éstas no se distribuyen por igual, y con los mismos valores, con todas las categorías gramaticales. Veámoslo en el siguiente cuadro:

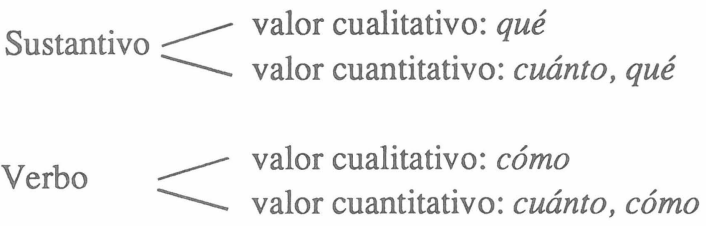

Adjetivo valor intensificativo: qué, (cuán)

Adverbio valor intensificativo: qué, (cuán)

Con sustantivos y verbos puede ocurrir que la correspondiente partícula ocupe el lugar funcional de un modificador «cualitativo» o «cuantitativo» y, en cada caso, la forma seleccionada será diferente. En principio, para el sustantivo funciona la oposición quélcuánto, y para el verbo la oposición cómo/cuánto. Ejemplos:

/12/a. ¡Qué chistes cuenta! (cualit.)

b. ¡Cuántos chistes sabe! (cuantit.)

/13/a. ¡Cómo se puso! (cualit.)

b. ¡Cuánto trabajan! (cuantit.)

Pero hay que hacer notar que el primer elemento de cada par opositivo (quéy cómo para $\mathrm{S}$ y $\mathrm{V}$, respectivamente), que consideramos el elemento no marcado de la oposición, tiene virtualidad para expresar también un valor cuantitativo, en ciertos contextos. Ejemplos como los de a. y b. pueden considerarse de significado similar en /14/y en /15/:

/14/a. ¡Qué pena me dio!

b. ¡Cuánta pena me dio!

/15/a. ¡Cómo os gusta el pescado!

b. ¡Cuánto os gusta el pescado! 
En relación con el verbo, I. Bosque ${ }^{17}$ indica que si se tratara de oraciones interrogativas no existiría la sinonimia:

/16/a. ¿Cómo os gusta el pescado?

b. ¿Cuánto os gusta el pescado?

Sin embargo, no hay que pensar que tal posibilidad de similitud significativa en la exclamación se da con todos los sustantivos y con todos los verbos. Tengamos ejemplos como los siguientes:

/17/a. ¡Cómo trabaja!

b. ¡Cuánto trabaja!

c. ¡Qué libros tienen!

d. ¡Cuántos libros tienen!

En el caso del verbo, $/ 17 \mathrm{a}$ / puede referirse, según el contexto, a la forma de trabajar o a la cantidad de trabajo que realiza. Para el sustantivo, $/ 17$ c/ tiene más posibilidades de ser interpretado como cualitativo. Todo ello quiere decir que la interpretación cuantitativa de qué y cómo dependerá, en cada caso de la significación particular del sustantivo o el verbo y de las condiciones del contexto en que se emite el enunciado.

La lengua dispone, además, de otro recurso sintáctico para expresar sin ambigüedades la «cantidad» con sustantivos. Se trata de la contrucción qué de, la cual nos recuerda un cierto valor «partitivo» de la preposición:

/18/a. ¡Qué de coches circulan por aquí!

b. ¡Qué de sandeces!

Volviendo sobre el cuadro antes expuesto, en las otras dos categorías gramaticales, adjetivo y adverbio, carece de pertinencia funcional la oposición «cualitativo»/ «cuantitativo» antes planteada. La forma que tales categorías seleccionan normalmente es qué, aportando un contenido «intensificativo» a la cualidad expresada en la correspondiente significación léxica:

/19/a. ¡Qué contento se puso!

b. ¡Qué lejos vive!

Habría que prestar atención a los tipos de adjetivos y de adverbios que pueden recibir la incidencia de la forma qué. Sólo la admiten aquellos cuyo significado puede ser graduado y, por tanto, intensificado. De una «casa grande», de un «problema difícil» o de un «niño alto» podríamos decir:

/20/a. ¡Qué grande es esta casa/

b. ¡Qué difícil es este problema!

c. ¡Qué alto se ha puesto el niño!

${ }^{17}$ I. Bosque: «La selección...», op. cit. 
Pero de una «carrera ciclista», de la «lingüística estructural» o de una «nueva fórmula química», difícilmente se darían construcciones como:

/21/a. ${ }^{i}$ Qué ciclista parece esta carrera!

b. *iQué estructural es esta lingüística!

c. *iQué química me resulta esta nueva fórmula!

Algo similar ocurre con los adverbios. Unos pueden recibir la intensificación y otros no:

$$
\begin{aligned}
& / 22 / \text { iQué }\left\{\begin{array}{l}
\text { bien/mal } \\
\text { lejos/cerca } \\
\text { fácilmente } \\
\text { temprano/tarde } \\
\ldots
\end{array}\right\} ! \\
& / 23 / *_{\text {iQué }}\left|\begin{array}{l}
\text { aquí } \\
\text { antes/después } \\
\text { casi } \\
\text { mientras } \\
\ldots
\end{array}\right| !
\end{aligned}
$$

Ahora bien, el estudio de los adjetivos y los adverbios que admiten o no la gradación no es un problema específico de las exclamativas, sino de la cuantificación en general. Los mismos que admiten qué en el grupo de /20/o de /22/ son los que pueden ser modificados por cuantificadores como muy, bastante, demasiado, etc. Por ello es una cuestión que merece tratamiento aparte ${ }^{18}$.

\section{2. Exclamativas con adjetivo ponderativo}

Hasta ahora hemos visto la tipología estructural de las «exclamativas $q u$-» según el tipo de partícula que selecciona cada categoría gramatical, pero se hace necesario atender, además, a otros criterios de configuración sintáctica.

Hay un tipo de enunciados que se caracterizan por la presencia de un adjetivo ponderativo acompañando al sustantivo. Dicho adjetivo puede ir precedido de cuantificadores como más o tan, o bien puede no llevarlos ${ }^{19}$ :

/24/a. ¡Angelito, qué susto más grande le hicieron pasar! (C. J. Cela)

b. ¡Qué tristeza tan honda!

${ }^{18}$ En lo referente a las posibilidades de cuantificación de ciertos adverbios, cf. P. Carbonero: Deíxis espacial y temporal en el sistema lingüístico. Sevilla, Publ. de la Universidad, 1979. Pág. 81.

${ }^{19}$ Son los «adverbios de encarecimiento» que menciona S. Fernández Ramírez, op. cit., pág. 506. 
/25/a. Qué paz estéril, solitaria, llena aquel vivir pasado en lontananza. (L. Cernuda)

b. ¡Qué sitio deprimente! ( $R$. Montero)

Estas estructuras raramente se darían en las interrogativas, porque el adjetivo da respuesta a la hipotética incertidumbre del hablante. Por ejemplo:

/26/a. (Pregunta) ¿Qué sitio?

b. (Respuesta) Un sitio deprimente.

En $/ 25 b /$, sin embargo, la posible respuesta va incluida en el propio enunciado. Se trata, pues, de exclamaciones de base nominal, en que la partícula exclamativa incide sobre el sustantivo, pero hay un adjetivo que lo califica, aportando un juicio afectivo, valorativo.

Hemos podido observar, por otra parte, que las estructuras sin el llamado «adverbio de encarecimiento» son más habituales en el lenguaje poético o literario, frente a las que lo poseen, que participan más a menudo del estilo coloquial.

\subsection{Estructuras bimembres}

Como se ha venido apreciando a través de los ejemplos, los enunciados exclamativos no siempre han de tener estructura oracional, es decir, no es necesario que estén dotados de un núcleo verbal predicativo en forma finita para que tengan lo que suele llamarse «sentido completo». Así ocurre, por ejcmplo, en /18b/,/24b/o/25b/.Pues bien, en tal caso se detectan dos posibles estructuras: unimembres (como las de $/ 24 \mathrm{~b} / \mathrm{y}$ /25b/) o bimembres (como las de /26/):

/26/a. Qué ruido tan triste el que hacen dos cuerpos cuando se aman (L. Cernuda)

b. ¡Ay, qué acontecimiento de quebranto ir a tu corazón y hallar un hielo de irreductible y pavorosa nieve! (M. Hernández)

c. Mirad qué oscuro se pone el chorro de la montaña.

¡Ay, qué blanca

la triste casada! (F. García Lorca)

En estos ejemplos, el sintagma que sirve de base a la exclamación -y que nosotros hemos subrayado- es el predicado (no verbal) y el segundo miembro del enunciado es el sujeto, el cual puede adquirir estructuras diversas: oración de relativo nominalizada (en /26a/), oración de infinitivo (en /26b/), sintagma nominal (en /26c/), etc. 
Este aspecto está conectado con el que estudia I. Bosque ${ }^{20}$ al contrastar construcciones del tipo:

/27/a. ¡Qué bien que cocina María!

b. ¡Qué bien que cocine María!

A pesar de la similitud formal hay evidentes diferencias entre una y otra. En enunciados como /27a/ la forma que es opcional. Su elisión no impide que se mantenga la misma estructura oracional, ya que el adverbio que sirve de base a la exclamación seguiría modificando al verbo de la misma manera. Otros ejemplos, con base nominal 0 adjetiva pueden ser:

/28/a. ¡Qué libros (que) lee Antonio!

b. ¡Qué guapa (que) estás!

Por el contrario, en /27b/ el elemento que es obligatorio:

/29/ * QQué bien cocine María!

Pero este no es más que un ejemplo de la diversidad que pueden adquirir las estructuras bimembres, en las cuales el único rasgo generalizado de su configuración es que el segundo segmento, en el orden lineal, es un elemento nominal o nominalizado, del cual se predica el primero, el portador de la exclamación:

130/a. ¡Qué raro que me llame Federico!

b. ¡Qué raro, ese nombre!

c. ¡Qué raro, lo que me han contado!

Por ello /27b/ o /30a/ no pueden perder la forma que, la cual funciona como nominalizadora de la oración que introduce.

\subsection{Estructuras epitéticas}

Llamamos así a determinadas construcciones del tipo $N$ de $N$ en las que el primer $\mathrm{N}$ (nombre) indica una atribución epitética del segundo:

/31/a. ¡Qué asco de sopa!

b. ¡Qué maravilla de mujer!

c. ¡Qué miseria de sueldo!

d. ¡Qué vergüenza de reunión!

${ }^{20}$ I. Bosque: «Sobre la sintaxis...», op. cit. 
En ellas subyace una estructura atributiva como:

/32/a. Esta sopa es un asco.

b. Esa mujer era una maravilla.

c. El sueldo es una miseria.

d. La reunión fue una vergüenza.

Como correlativas de las anteriores, también podrían darse estructuras epitéticas no exclamativas. Así:

133/a. Es un asco de sopa.

b. Era una maravilla de mujer.

c. Es una miseria de sueldo.

d. Fue una vergüenza de reunión.

Nos encontramos aquí con una construcción que no es exclusiva de los enunciados exclamativos y que ha sido ampliamente estudiada en el dominio románico ${ }^{21}$, según el modelo ce fripon de valet. Hay sintagmas nominales en los que el primer elemento puede ser un adjetivo sustantivado (el tonto del niño, el feo de Luis...) o un sustantivo (un asco de sopa, una birria de sueldo...). Y en este caso el primer sustantivo adquiere un carácter abstracto, valorativo, mientras que el segundo posee valor designativo, referencial. Y aunque señala $R$. Lapesa ${ }^{22}$ que no faltan grupos en que los dos componentes son abstractos, como en el ejemplo de J.R. Jiménez ¡Qué tristeza de olor de jazmín!, no hay duda de que lo que se designa es el «olor de jazmín».

Pero no todos estos sintagmas pueden convertirse en enunciados exclamativos, sino sólo aquellos que, como tal construcción epitética son susceptibles de aparecer como atribuciones de carácter valorativo del tipo $/ 33 /$. Obsérvese que el ejemplo de $/ 34 \mathrm{a} /$ (que se construye como los de /32/) no puede transformarse en $/ 34 \mathrm{~b} /$ (donde toda la construcción epitética funcionaría como atribución) y, por consiguiente, tampoco en /34c/. Lo mismo ocurre en /35/:

134/a. El niño es un tonto.

b. *Es un tonto de niño.

c. *iQué tonto de niño!

135/a. El profesor es un hueso.

b. *Es un hueso de profesor.

c. ${ }^{*}$ iQué hueso de profesor!

${ }^{21}$ Cf. R. Lapesa: «Sobre las construcciones El diablo del toro, El bueno de Minaya, jAy de mí!, jPobre de Juan!, Por malos de pecados», Filología, 8 (1962), 169-184; M. Tutescu: «Le type nominal ce fripon de valet», Revue de Linguistique Romane, 33 (1969), 299-316; E. Alarcos: «Grupos nominales con /de/ en español», Studia Hispanica in Honorem R. Lapesa, I, Madrid, Gredos, 1972, 85-91; S. Gutiérrez Ordónez: «Grupos nominales N de N», Estudios ofrecidos a E. Alarcos Llorach, III, Oviedo, 1978, 133-159.

${ }^{22}$ R. Lapesa: «Los casos latinos: restos sintácticos y sustitutos en español», BRAE, 44 (1964), 57-105. 
Tales restricciones están relacionadas con la necesaria presencia del artículo ante el segundo nombre de las construcciones epitéticas correspondientes a ejemplos como los de /34/ o /35/ (el tonto del niño, el hueso del profesor) y su ausencia en las correspondientes a /33/ (un asco de sopa, una vergüenza de reunión... $)^{23}$.

\subsection{Otras formas ponderativas}

Existen unos elementos ponderativos, como menudo, valiente o vaya, que no son propiamente formas qu-, pero que parecen equivaler a ellas. En construcciones como las de /36/ podrían ser sustituibles por la exclamativa qué:

136/a. ¡Menudos tirantes lleva!

c. ¡Valiente personaje!

d. ¡Vaya gente!

Pero se dan entre ellas algunas diferencias:

$\left.1^{a}\right)$ Mientras que menudo y valiente sólo inciden sobre sustantivos, vaya puede acompañar, además, a adjetivos y adverbios:

137/a. ¡Vaya calor que hace!

b. ¡Vaya tonto que es!

c. ¡Vaya mal que lo hace!

$\left.2^{\underline{a}}\right)$ Realmente vaya no ocupa el mismo lugar funcional que qué, por cuanto puede coexistir con ella, así como con las otras formas qu-:

138/a. ¡Vaya qué listo es!

c. ¡Vaya cuánto dinero gana!

d. ¡Vaya cómo ha disfrutado!

Asimismo, la forma ponderativa vaya permite la presencia de determinantes o presentadores en el sintagma nominal:

139/a. ¡Vaya ese tío!

b. ¡Vaya una gente que vive allí!

Existe, por otra parte, la construcción ponderativa vaya si, que permite transformar los enunciados de /38/ en otros como los de /40/:

/40/a. ¡Vaya si es listo!

b. ¡Vaya si gana dinero!

c. ¡Vaya si ha disfrutado!

${ }^{23}$ Esta cuestión ha sido estudiada por A. Benito: El sustantivo como incremento prepositivo de otro sustantivo. Tesis Doctoral mecanografiada, Universidad de Sevilla, 1985. 
$3^{a}$ ) Con menudo y valiente se da, al igual que con las formas $q u$-, la posibilidad de un que demarcador, similar al que veíamos en /28/, con carácter opcional:

141/a. ¡Menudo carácter (que) tiene!

b. ¡Valiente elemento (que) está hecho!

Por el contrario, con vaya tal elemento que adquiere carácter casi obligatorio. Así se ve en los ejemplos de $/ 37 /$, los cuales difícilmente podrían enunciarse como:

142/a. * ¡Vaya calor hace!

b. ${ }^{*}$ iVaya tonto es!

c. ${ }^{*}$ iVaya mal lo hace!

Ello no significa que sea totalmente imposible encontrar algún ejemplo como:

/43/ ¡Vaya suerte tiene!

Pero construcciones como ésta son aisladas y no muy habituales.

\section{Enunciados sin partícula exclamativa}

Vamos a estudiar otras clases de enunciados, que se caracterizan por no estar introducidos por una forma $q u$ - ni por ninguna de las otras formas ponderativas consideradas hasta ahora. En este gran grupo puede apreciarse una gran variedad de construcciones, algunas de las cuales merecen especial atención por su frecuencia o por sus peculiaridades, según veremos a continuación. La mayor parte de ellas tienen la apariencia de estructuras «truncadas», que mantienen íntimas conexiones formales con las oraciones de relativo (ej.: jel dolor que tenía!), las comparativas (ej.: jera más huraño!), las consecutivas (ej.: jtodo venía tan sobre ruedas!) o las condicionales (ej.: isi vienes sudando!), a las que parece faltar un miembro de la construcción.

\subsection{Exclamativas y relativas}

Ya señala J.C. Milner ${ }^{24}$ algunos ejemplos de oraciones que, si se enunciaran como declarativas, parecerían tener un sintagma nominal al que le falta una oración de relativo que complete su significado. Y, por tanto, sin ella son interpretables como exclamativas. Así ocurre en:

/44/a. Tiene unos modales que no me gustan nada.

b. ¡Tiene unos modales!

${ }^{24}$ J. C. Milner: De la syntaxe..., op. cit., 325-326. 
Sin embargo, no hay que pensar que el segmento «ausente» sea necesariamente una oración. Podría ser cualquier otro tipo de adjetivación:

145/a. Comen unas cosas rarísimas.

b. Comen unas cosas de poco alimento.

c. ¡Comen unas cosas!

En cualquier caso, está claro el contenido modal exclamativo de /44b/ o de /45c/ por su equivalencia significativa con exclamativas $q u$ - como:

/46/a. ¡Qué modales tiene!

b. ¡Qué cosas comen!

Existe, por otro lado, un tipo de construcciones que parecen ser similares a las relativas y que a veces han merecido especial atención en algunos trabajos ${ }^{25}$, al presentar unos rasgos sintácticos peculiares y controvertidos:

/47/a. ¡La ropa que lleva!

b. ¡El dinero que tiene!

c. ¡Lo contenta que se ha puesto!

d. ¡Lo que disfrutaron aquel día!

e. ¡Lo lejos que vive!

En todas ellas se observa la estructura artículo... que y puede tener como base cualquiera de las categorías gramaticales: sustantivo (como en $/ 47 \mathrm{a} /$ y $/ 47 \mathrm{~b} /$ ), adjetivo (como en $/ 47 \mathrm{c} /$ ), verbo (como en $/ 47 \mathrm{~d} /$ ) o adverbio (como en $/ 47 \mathrm{e} /$ ). Pero el artículo requiere concordancia con el sustantivo, y no así con las otras categorías, para las cuales se usa ivariablemente la forma $l o$.

Está claro que tales construcciones no se dan exclusivamente en enunciados exclamativos simples, sino que pueden aparecer incorporadas en estructuras más complejas:

/48/a. Luis se asombró de los libros que habían leído los estudiantes.

b. No sabes los problemas que me has ocasionado con esto.

c. Le critican lo presumida que es.

d. No se imagina Vd. lo pronto que desapareció.

E. Alarcos señala al respecto que «el artículo/lo/ no sólo efectúa una determinación sino que añade una como estimación o gradación implícita, lo cual, por otra parte, es propio también de los otros artículos». ${ }^{26}$ Este autor alude también a A. Bello ${ }^{27}$, quien observa este matiz de tipo enfático en lo que.

${ }^{25}$ E. Alarcos: «Lo fuertes que eran», en Estudios de gramática funcional del español. Madrid, Gredos, 1984; S. Plann: «Cláusulas cuantificadas», Verba, 11 (1984), 101-129.

${ }^{26}$ E. Alarcos: «Lo fuertes que eran», op. cit., pág. 189.

${ }^{27}$ A. Bello: Gramática de la lengua castellana. Buenos Aires, Sopena, 1970. 
S. Plann indica que algunas de estas estructuras pueden ser interpretadas como «cláusulas restrictivas» o como «cláusulas cuantificadas», según los contextos en que aparecen, y explica que cuando son interpretadas como cláusulas cuantificadas se entiende que contienen un cuantificante no realizado ${ }^{28}$. Con ello quiere decir que ejemplos como los de /48a/ o /48b/ serían equivalentes a:

149/a. Luis se asombró de los muchos libros que habían leído los estudiantes.

b. No sabes la cantidad de problemas que me has ocasionado con esto.

Sin embargo, creemos que la interpretación no siempre ha de ser cuantitativa, sino que puede ser cualitativa. Así, cabría entender $/ 48 \mathrm{a} /$ como $/ 49 \mathrm{a} /$, pero también como 150/:

/50/ Luis se asombró de la clase de libros que habían leído los estudiantes.

El que se dé una u otra interpretación dependerá del significado de la frase y del contexto en que ocurra. Si buscamos una correlación con las exclamativas $q u-, / 47 \mathrm{a} /$ puede ser transformada en $/ 51 \mathrm{a} /$, pero /47b/ lo es en $/ 51 \mathrm{~b} /$ :

151/a. ¡Qué ropa lleva! (cualidad)
b. i $\left\{\begin{array}{l}\text { Cuánto } \\ \text { Qué (de) }\end{array}\right\}$ dinero tiene!
(cantidad)

Cuando no hay duda de que adquiere una interpretación cuantitativa es si aparece la construcción la de + sustantivo + que, donde el artículo tiene la forma invariable de femenino singular y que es correlativa de la exclamación con qué de (que ya vimos en ejemplos como/18/). Así:

152/a. ¡La de chistes que sabe!

b. ¡La de coches que circulan por aquí!

Por otra parte, S. Plann ha observado acertadamente que, cuando son interpretables como cláusulas cuantificadas, se trata de estructuras de núcleo verbal, a diferencia de las relativas restrictivas, que son de núcleo nominal. Algunas pruebas de ello son las siguientes:

a) El complemento directo de verbos como saber o averiguar no suele ser un sintagma nominal con sustantivo de persona, pues se producirían expresiones inaceptables:

153/a. No sabes los novios que ha tenido Lola.

b. *No sabes los novios.

c. Papá va a enfadarse cuando averigüe los amigos que tienes.

d. *Papá va a enfadarse cuando averigüe los amigos.

${ }^{28}$ S. Plann: «Cláusulas cuantificadas», op. cit. 
b) No suele haber concordancia entre el verbo principal y el sustantivo, como sería propio si éste fuera el núcleo del sujeto. Una oración como:

154/Es asombroso las historias que cuenta.

es plenamente aceptable y las historias no concuerda con es ni con asombroso. Esto indica que el sujeto de dicho verbo es todo el segmento las historias que cuenta, al que hay que atribuir carácter de estructura oracional donde la proposición «sabe (muchas) historias» está sustantivada por la forma artículo... que, que le sirve de traspositor.

c) Tampoco la presencia de un pronombre anafórico suele requerir concordancia con el sustantivo:

155/a. Cuando me dijeron los días que estuvieron sin comer, no me lo puede creer.

b. *Cuando me dijeron los días que estuvieron sin comer, no me los pude creer.

En consecuencia, se trata de construcciones de núcleo verbal, que al incorporarse como subordinadas en una estructura superior sufren una trasposición sustantiva.

Hay una circunstancia especial que conviene considerar también, y es la presencia de ciertas preposiciones. Cuando una exclamativa $q u$ - tiene como base un sustantivo, la partícula va precedida de la preposición que corresponda a la función sintáctica del sintagma nominal. Ejemplos:

156/a. ¡Con cuánta pena me lo dijo!

b. ¡A cuántos países ha viajado!

Y por ello, paralelamente, la preposición puede preceder a la forma artículo... que:

157/a. ¡Con la pena que me lo dijo!

b. ¡A $\left\{\begin{array}{l}\text { los } \\ \text { la de }\end{array}\right\}$ países que ha viajado!

Algunos autore ${ }^{29}$ han visto en ello una anteposición potestativa de la preposición, procedente de estructuras como:

158/a. ¡La pena con que me lo dijo!

b. ¡Los países a los que ha viajado!

Pero, si aceptamos el carácter de nuclearidad verbal en las construcciones de /57/, no hay que ver nada extraño en que la preposición preceda a la pena para complementar a lo dijo, o a los países para complementar al verbo ha viajado. Sin embargo, no se deben confundir estructuras como las de /57/ con otras, como las de /59/, a pesar de su aparente similitud:

${ }^{29}$ S. Gili Gaya: Curso superior de sintaxis española. Barcelona, Vox, 1983, pág. 305. 
159/a. ¡Con lo cansado que estoy!

b. ¡Con la fuerza que parecía tener!

ya que ni el sintagma lo cansado ni la fuerza requieren la preposición para complementar, respectivamente, a los núcleos verbales estoy o parecía tener, al desempeñar funciones sintácticas como la de atributo o de complemento directo. Por ello sería imposible el cambio de lugar de la preposición para anteponerse inmediatamente a la forma que.

En $/ 59 /$, por tanto, la preposición introduce a todo el resto del enunciado, y no exclusivamente al sintagma nominal, dotando a la exclamación de matices significativos como el de cierto valor causal, en $/ 59 \mathrm{a} /$, o adversativo, en $/ 59 \mathrm{~b} /$, ya que permiten prolongaciones como:

160/a. Con lo cansado que estoy, creo que me volveré temprano a casa.

b. Con la fuerza que parecía tener, no fue capaz de derribarlo.

\subsection{Exclamativas, consecutivas y comparativas}

También se da una similitud entre las exclamativas y ciertas oraciones consecutivas y comparativas con el carácter de «truncadas», esto es, que carecen del segundo miembro de la correlación. Compárense, por ejemplo:

161/a. ¡Era tan fácil todo!

b. Era tan fácil todo, que lo terminé antes de lo previsto.

Por su parte, construcciones como las de /62/ admiten dos tipos de análisis: $:^{30}$

/62/a. Hace más calor en Sevilla.

b. Trabaja menos.

Podrían considerarse comparativas si llevan entonación declarativa y se enuncian integradas en contextos como los de /63/:

163/a. Aquí hace calor, pero hace más calor en Sevilla.

b. Antonio trabaja menos que Luis.

Pero fuera de tales contextos suelen ser interpretadas como exclamativas simples, sobre todo si están dotadas de la entonación correspondiente. Volveremos sobre ellas en el apartado siguiente. 


\section{Sinopsis}

Ante la variedad de configuraciones que pueden adquirir los enunciados exclamativos, según se ha estudiado en los apartados anteriores, nos parece necesario elaborar una sinopsis que muestre de manera global y sistemática las posibilidades y restricciones de que dispone la lengua en este campo. Por razones de claridad expositiva, podemos agrupar los enunciados en tres tipos:

1) El primer grupo está constituido por las construcciones dotadas de partícula exclamativa (cf. ap. 1).

2) Un segundo grupo es el que se basa en la forma el... que, lo... que, etc. (cf. ap. 2.1.).

3) En el tercero de ellos reunimos aquellas estructuras «truncadas» que tienen similitud formal con las adjetivas, las consecutivas y las comparativas, así como ciertas construcciones «partitivas», dotadas de la preposición de precediendo a sustantivos, adjetivos o adverbios, como en los siguientes casos:

164/a. ¡Compró de regalos!

b. ¡Está de loco!

c. ¡Vive de bien!

Las posibilidades de estructuración gramatical y las correlaciones entre los tres grupos quedan representados en el cuadro de la página siguiente ${ }^{31}$.

En este cuadro se especifican de forma sintética las configuraciones que pueden adquirir los enunciados para expresar, respectivamente, la exclamación de valor «cualitativo», «cuantitativo» e «intensificativo» (valores que ya fueron explicados en el apartado 1.1.). Como ya se vio, la distinción «cualitativo»/«cuantitativo» es aplicable a las que tienen como base un sustantivo $(\mathrm{N})$ o un verbo $(\mathrm{V})$, mientras que el rasgo «intensificativo» es aplicable a adjetivos (Adj.) y adverbios (Adv.). A su vez, si la base es un sustantivo, es pertinente -para ciertas restricciones- distinguir si éste va o no precedido de preposición. Cuando existe una preposición, en el cuadro se representa la inicial P. Además, el subíndice $a$ o $b$ sirve para diferenciar dos tipos de contextos:

a) Se aplica este subíndice a las exclamativas $\mathrm{N}, \mathrm{V}$ o $\mathrm{P}$ en las que el sustantivo o el verbo puede ser cuantificable o cualificable y que, por tanto, requieren configuraciones diferenciadas si se intenta dar una u otra interpretación. Véase, por ejemplo, la diferencia significativa entre /65/ y /66/:

/65/a. ¡Qué colores le ha puesto!

b. ¡Le ha puesto unos colores! (cualit.)

/66/a. ¡Qué de colores le ha puesto!

b. ¡Le ha puesto de colores!

(cuantit.)

${ }^{31}$ Para mayor claridad indicamos con una numeración a la derecha de cada forma los ejemplos que pueden servir de ilustración. 


$$
\mathbb{1}^{\text {er }} \text { grupo } \quad 2^{\text {o }} \text { grupo } \quad 3^{\text {er }} \text { grupo }
$$

$\mathrm{N}_{\mathrm{a}} \quad q u e ́$ /65a/ el..que $/ 47 \mathrm{a} /$ un $/ 65 \mathrm{~b} /$

Cualit.

$\begin{array}{lccc}\mathrm{V}_{\mathrm{a}} & \text { cómo } 71 \mathrm{a} / & - & \text { (un) } 71 \mathrm{~b} / \\ \mathrm{P}_{\mathrm{a}} & \text { qué } 770 \mathrm{~b} / & \text { el...que } / 57 \mathrm{~b} / & \text { un } / 70 \mathrm{a} /\end{array}$

$\mathrm{N}_{\mathrm{a}} \quad$ cuánto $/ 74 \mathrm{c} /$ el...que /48a/ más $/ 74 \mathrm{a} /$
qué de $/ 18 \mathrm{a} / \quad$ lade...que /52a/ de $774 \mathrm{~b} /$

\begin{tabular}{|c|c|c|c|}
\hline $\mathrm{N}_{\mathrm{b}}$ & $\begin{array}{l}\text { cuánto /14b/ } \\
\text { qué / 67a/ } \\
\text { (qué de) /68a/ }\end{array}$ & el...que /47b/ & $\begin{array}{l}\text { más } / 75 \mathrm{a} / \\
\text { un } / 67 \mathrm{~b} / \\
(d e) / 68 \mathrm{~b} /\end{array}$ \\
\hline
\end{tabular}

$\mathrm{V}_{\mathrm{a}} \quad$ cuánto $/ 78 \mathrm{c} / \quad$ - más $/ 78 \mathrm{a} /$

Cuant.

$\mathrm{V}_{\mathrm{b}} \quad \begin{aligned} & \text { cuánto } / 15 \mathrm{a} / \\ & \text { cómo } / 15 \mathrm{~b} /\end{aligned}$ lo...que $/ 47 \mathrm{~d} / \quad \begin{aligned} & \text { más } / 78 \mathrm{a} / \\ & \text { (un })\end{aligned}$

$\begin{array}{lll}\mathrm{P}_{\mathrm{a}} & \text { cuánto } / 79 \mathrm{cl} & \text { ęl...que } / 57 \mathrm{~b} / \\ & \text { qué de } / 66 \mathrm{a} / & \text { la de...que } / 57 \mathrm{~b} /\end{array}$
$\mathrm{P}_{\mathrm{b}}$
cuánto /56a/
qué /80c/
el. . que 157a/
más /80a/ un $773 \mathrm{a} /$

Adj.

qué $776 \mathrm{c} /$

lo. . que /47 c/

más $776 \mathrm{a} /$ (cuán) de $776 \mathrm{~b} /$

Intens.

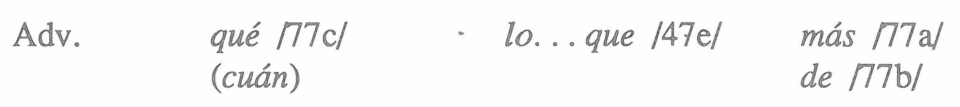


b) Este subíndice se aplica a las exclamativas N, V o P en las que el sustantivo o el verbo sólo suele admitir una interpretación cuantitativa y, por consiguiente, se neutraliza la distinción anterior. Obsérvese, por ejemplo, la similitud significativa entre /67/ y /68/, aunque presentan la misma configuración que /65/ y /66/, respectivamente.

167/a. ¡Qué frío hace!

b. ¡Hace un frío!

168/a. ¡Qué de frío hace!

b. ¡Hace de frío!

Conviene que nos detengamos un poco en explicar algunos aspectos correspondientes a las formas del tercer grupo. Se trata de conocer las posibilidades de aparición de cada uno de los tres tipos que rotulamos abreviadamente como un (ej. $/ 69 \mathrm{a} /$, más (ej. $74 \mathrm{a} /$ ) y de (ej. $/ 74 \mathrm{~b} /)$. Según se observa en el cuadro, las construcciones con más y con un presentan, en principio, una distribución complementaria: un para el valor cualitativo, y más para el cuantitativo. Adviértase, por su parte, que las exclamativas con un se dan sólo con sustantivos, lleven o no preposición:

169/a. ¡Comen unas cosas!

b. ¡Qué cosas comen!

70/a. ¡ Viaj6 a unos países!

b. ¡A qué países viajó!

Pero cuando se trata de exclamativas cualitativas de base verbal -equivalentes a las construcciones con cómo- la forma con un sólo es posible si dotamos al enunciado de un apoyo nominal, como en:

711/a. ¡Cómo se explica!

b. ¡Se explica de una manera!

Otro aspecto a señalar es que un puede aparecer en la exclamación cuantitativa, pero sólo en los casos en que se produce la neutralización de rasgos ya explicada para los grupos $\mathrm{N}_{\mathrm{b}}, \mathrm{V}_{\mathrm{b}}$ y $\mathrm{P}_{\mathrm{b}}$. Ejemplos:

772/a. ¡Hace un (a) calor!

b. i $\left\{\begin{array}{l}\text { Qué } \\ \text { Cuánto }\end{array}\right\}$ calor hace!

773/a. ¡Me lo dijo con una pena!

b. ¡ Con $\left\{\begin{array}{l}\text { qué } \\ \text { cuánta }\end{array}\right\}$ pena me lo dijo!

Centrándonos ahora en el valor «cuantitativo» e «intensificativo» -que a los efectos de distribución que estamos considerando constituyen un sólo grupo de 
funcionamiento-, se observará en el cuadro que la forma más es válida para todos los casos, mientras que la forma de se ve restringida a las exclamaciones con base sustantiva, adjetiva o adverbial; y no se da en las que tienen base verbal ni en las que el sintagma nominal lleva preposición. Si lo ilustramos con ejemplos, se observará que la forma de coexiste con más en $/ 74 /\left(\mathrm{N}_{\mathrm{a}}\right), / 75 /\left(\mathrm{N}_{\mathrm{b}}\right), / 76 /$ (Adj.) y $77 /$ (Adv.) (aunque, en algunos contextos, construcciones como $/ 75 \mathrm{~b} /$ pueden parecer extrañas o poco habituales), pero no puede alternar con más en $/ 78 /(\mathrm{V})$, en $/ 79 /\left(\mathrm{P}_{\mathrm{a}}\right)$ o en $/ 80 /\left(\mathrm{P}_{\mathrm{b}}\right)$, donde originaría una configuración sintáctica ajena a las reglas de construcción de nuestra lengua:

774/a. ¡Compró más regalos!

b. ¡Compró de regalos!

c. ¡Cuántos regalos compró!

775/a. ¡Tiene más fuerza!

b. ¡Tiene de fuerza!

c. ¡Qué fuerza tiene!

776/a. ¡Está más loco!

b. ¡Está de loco!

c. ¡Qué loco está!

777/a. ¡Se ha levantado más temprano!

b. ¡Se han levantado de temprano!

c. ¡Qué temprano se ha levantado!

778/a. ¡Se reían más!

b. * iSe reían de!

c. ¡Cuánto se reían!

179/a. ¡Han suspendido a más alumnos!

b. ${ }_{i}{ }_{i} H a n$ suspendido a de alumnos!

c. ¡A cuántos alumnos han suspendido!

/80/a. ¡Lo dijo con más soltura!

b. ${ }^{*}$ iLo dijo con de soltura!

c. ¡Con qué soltura lo dijo!

Todas las posibilidades gramaticales estudiadas en este grupo quedan representadas, pues, en el siguiente diagrama de rasgos binarios: 


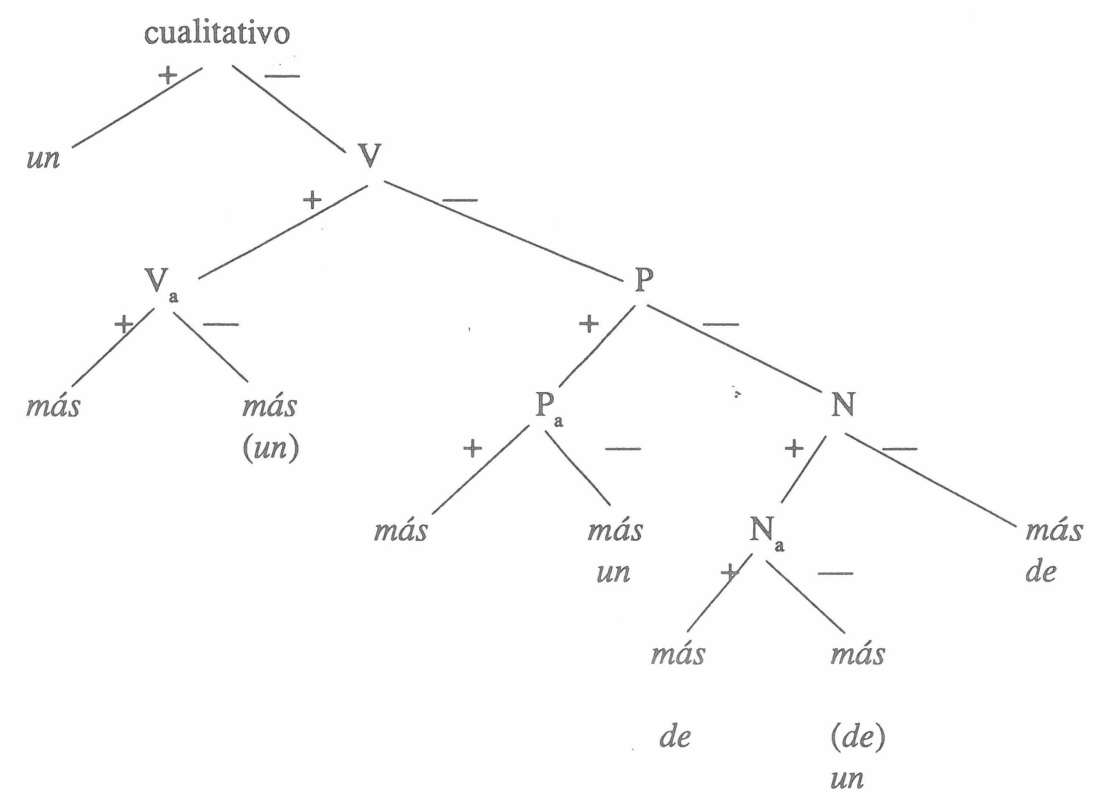

Hemos de aclarar que en cuadro no aparece representada la forma tan(to), pero que sus posibilidades de aparición coinciden con las de más. Sin embargo, entre una y otra forma de exclamación no se da plena coincidencia significativa. Tengamos ejemplos como los siguientes:

/81/a. ¡Qué insoportable me resulta!

b. ¡Cuánto tardan en llegar!

182/a. ¡Me resulta más insoportable!

b. ¡Tardan más en llegar!

/83/a. ¡Me resulta tan insoportable!

b. ¡Tardan tanto en llegar!

Si bien las construcciones de $/ 82 /$ (con más) representan el mismo significado que las de /81/ (con formas $q u$-), en /83/ (con $\tan ($ to $)$ ) cabe percibir un matiz de «justificación» añadida, según el cual el hablante suele emitir este tipo de enunciados cuando necesita aclarar sus actitudes o comentarios previamente evidenciados ante su interlocutor. 


\section{Otras estructuras}

La diversidad de construcciones con que se puede expresar la exclamación no se agota con las que hemos estudiado hasta aquí. Es preciso reconocer la existencia de otras posibilidades de configuración, de las que sólo vamos a presentar una muestra abierta, indicativa de la riqueza expresiva que es propia del lenguaje afectivo:

a) Oraciones independientes con si. Este tipo de oraciones, que no siempre implican valor exclamativo, ya han sido objeto de algunos estudios específicos ${ }^{32}$. He aquí algunos ejemplos:

/84/a. ¡Si es usted la mujer de la suerte, hija!

b. ¡Si vienes sudando!

b) Dicterios y requiebros. S. Fernández Ramírez ${ }^{33}$ da esta denominación a ciertos sintagmas nominales que se diferencian del vocativo en que «se despojan de su intención de llamamiento y se concentran en una declaración directa de carácter cualificante». Hay que observar en ellos que los sustantivos tienen un significado nítidamente valorativo:

185/a. ¡Partida de granujas!

b. ¡Atajo de maleantes!

c) Las construcciones con la forma qué $+N+n i q u e ́$ + frase hecha indican en su significado un rechazo o negación de lo «afirmado» o «presupuesto» por el oyente:

/86/a. ¡Qué timidez ni qué ocho cuartos!

b. ¡Qué rey ni qué niño muerto!

Otras, con la forma ni que + oración denotan más bien un rechazo o negación de lo «pretendido» por el oyente:

/87/ ¡Ni que yo fuera el oráculo!

d) La construcción mira que + infinitivo suele emplearse para introducir oraciones en que el hablante manifiesta reproche o sorpresa por algo ocurrido inesperadamente:

/88/a. ¡Mira que perder las llaves!

b. ¡Mira que ponerse a llover ahora!

${ }^{32}$ L. Contreras: «Oraciones independientes introducidas por "si"», Boletín de Filología de la Universidad de Chile, 12 (1960), 273-290; R. Almela: «El si introductor de oraciones independientes en español», Lingüística Española Actual, 7,1 (1985), 5-13.

${ }^{33}$ S. Fernández Ramírez, op. cit., pág. 498. 
Por su parte, cuando una forma que no acentuada precede a la oración, ésta suele indicar sorpresa o reproche por estar ocurriendo o a punto de ocurrir algo que el hablante no espera o no desea:

/89/a. iQue me ahogo!

b. ¡Que se va a volar el sombrero!

\section{Exclamación y negación}

La negación en los enunciados exclamativos presenta unos caracteres especiales. Como ya apreciaron A. Culioli ${ }^{34}$ y J.C. Milner, hay contextos en que puede aparecer libremente una forma negativa que no hace más que intensificar el valor afirmativo de lo expresado:

190/a. ¡No es tonto!

b. ¡No eran duras esas correas!

Por el contrario, se puede indicar un valor negativo o de rechazo sin ninguna forma expresa de negación:

191/a. ¡Qué voy a saber!

b. ¡Tres horas para hacer esto!

Y es que, en sentido esctricto, la exclamación, por su significado afectivo, difícilmente puede ser negada, o al menos no lo es de la misma manera que las aserciones. No obstante, sería posible encontrar ciertos recursos capaces de expresar un contenido similar a la negación. Entre ellos están:

a) Conmutación de qué o cuánto por qué poco ( $1^{\mathrm{er}}$ grupo):

192/a. ¡Cuánto te favorece ese vestido!

b. $\mathrm{Q}$ Qué poco te favorece ese vestido!

193/a. ¡Qué calma!

b. ¡Qué poca calma!

b) Conmutación de lo... que por lo poco... que (2º grupo):

194/a. ¡Los goles que metieron!

b. Los pocos goles que metieron!

195/a. jLo que me gusta esa chica!

b. ¡Lo poco que me gusta esa chica!

${ }^{34}$ A. Culioli, op. cit. 
c) Conmutación de más por menos (3er grupo):

196/a. ¡Tiene más imaginación!

b. ¡Tiene menos imaginación!

d) Conmutación de la base léxica de la exclamación por su antónimo:

197/a. ¡Qué ilusionado se te ve!

b. ¡Qué desilusionado se te ve!

198/a. ¡Qué hábilmente actuó!

b. ¡Qué torpemente actuó!

Este último recurso dependerá lógicamente de las posibilidades léxicas de la lengua, pues no siempre existe o es usual un antónimo correspondiente a cada vocablo.

\section{Teoría de la exclamación}

Tras la descripción de los hechos lingüísticos, hay que llegar, dando un paso más, a la explicación de los mismos. A partir de las construcciones analizadas en nuestro trabajo, debemos proponer una teoría explicativa que nos ayude a conocer la naturaleza de los enunciados exclamativos.

Una de las perspectivas adoptadas en el análisis de los enunciados no-declativos por $\mathrm{R}$. Zuber ${ }^{35}$ es la de estudiar su correspondencia y relación con los declarativos correspondientes. Así, podría decirse que /99b/ es correlativa de la declarativa /99a/, si se le aplica la marca [+interrogación]:

199/a. Los alumnos han fijado la fecha del examen.

b. ¿Han fijado los alumnos la fecha del examen?

Pero, cuando se trata de una forma $q u$-, ¿podría decirse que /100b/ corresponde a /100a/, a la que se ha dotado de una marca de [+exclamación]?:

/100/a. Esa película es violenta.

b. ¡Qué violenta es esa película!

Creemos que tal correspondencia es sólo aparente. Porque, de ser así, habría enunciados declarativos que no tienen su correlato exclamativo:

/101/a. Mi amigo vive en las afueras de la ciudad.

b. *iQué en las afueras de la ciudad vive mi amigo!

${ }^{35}$ R. Zuber: Non-declarative Sentences. Amsterdam, John Benjamins Publ., 1983. 
Las exclamativas qu- (así como las interrogativas del mismo tipo) deben entenderse como estructuras sintagmáticas en las que un constituyente está «vacante», designativamente vacío, y su lugar está formalmente ocupado por una partícula. Por ello, para que una oración como /101a/ pueda convertirse en exclamativa, hay que proceder al vaciado de uno de sus constituyentes. Por ejemplo, eliminando el segmento en las afueras de la ciudad, obtendríamos una esctructura truncada como:

/102/ Mi amigo vie LOC.

donde queda un lugar estructural que representa la función LOC (locativo). La correspondiente exclamativa se obtiene haciendo ocupar el lugar vacío por una forma $q u$ - que indique dicho valor funcional. Y en este caso se trataría de la forma dónde, la cual, según una regla general, se traslada al principìo de la cadena sintagmática:

\section{/103/ ¡Dónde vive mi amigo!}

Además del «movimiento de $q u-»^{36}$ al primer lugar, el orden de otros elementos de la oración puede verse modificado, según las reglas que rigen tal ordenación en nuestra lengua (en este ejmplo, la posposición del sujeto mi amigo).

Así pues, la correspondiente declarativa de /100b/ no es /100a/, sino /104/, donde INT representa cualquier intensificador del tipo muy, bastante, tremendamente, etc.:

/104/ Esa película es INT violenta.

En/100b/el hablante deja sin expresar léxicamente la «intensidad de la violencia», igual como en /103/ no designa el «lugar donde vive mi amigo».

También hemos tenido ocasión de estudiar, a lo largo de este trabajo, ciertas exclamativas sin partícula, que se caracterizan por la ausencia de un miembro de la construcción, lo que le da la apariencia de estructura sintácticamente truncada.

Pero habría que preguntarse cuál es la explicación funcional, comunictiva, de ese vaciado designativo. La exclamación supone la imposibilidad que tiene el hablante de expresar algo en sus justos términos, pero no por desconocimiento, como en la interrogación, sino porque «no tiene palabras». Ello es consecuencia del cáracter afectivo que adquiere el enunciado procedente de «una emoción del ánimo, sea de alegría, pena indignación, cólera, asombro o cualquier otro afecto» ${ }^{37}$.

Ello está relacionado con la teoría del «alto grado» que proponen J.C. Milner o J. Gérard $^{38}$, según la cual la exclamación se sitúa en el punto más alto de una escala de gradación:

${ }^{36}$ N. Chomsky: «On Wh-movement», en P. Culicover, T. Wasow y A. Akmajian (eds.): Formal Syntax, New York, Academic Press, 1977, 71-132.

${ }^{37}$ Tomado de la definición de la voz exclamación, en R.A.E.: Diccionario de la Lengua Española. Madrid, Espasa-Calpe, 1984.

${ }^{38}$ J. Gérard, op. cit. 
/105/a. Hace frío.

b. Hace mucho frío.

c. Hace muchísimo frío.

d. ¡Qué frío hace!

Pero pensamos que a veces es difícil aceptar tal interpretación, por varias razones:

$\left.1^{\text {a }}\right)$ No siempre el «constituyente vacío» representa un grado (es decir, cuantificador o intensificador) como en /105/. En las que hemos llamado exclamativas «cualitativas», como la del ejemplo /106a/, lo que se omite designativamente no es un cuantificador sino un cualificador. Por eso su correlato no es /106b/, sino /106c/:

/106/a. ¡Qué palabras decía!

b. Decía muchas palabras.

c. Decía palabras $\left\{\begin{array}{l}\text { raras } \\ \text { extrañas } \\ \text { feas } \\ \text { desagradables... }\end{array}\right\}$

$2^{2}$ ) Hay situaciones contextuales que pueden favorecer la interpretación de un «grado menor». Por ejemplo, con la oración:

/107/¡Cómo come este niño!

según la entonación y el contexto, puede entenderse que «come mucho» o que «come poco», lo que permitiría prolongaciones sintagmáticas como la siguiente:

/108/iCómo come este niño! En cuanto se toma dos cucharadas, dice que ya no tiene ganas.

En definitiva, es la ausencia de determinados elementos léxicos específicos (de contenido cuantitativo o cualitativo), junto con los correspondientes rasgos de entonación, lo que da a los enunciados exclamativos esa fuerza expresiva con la que el hablante aporta al «dictum» un valor modal de juicio afectivo. Hay muchas maneras de manifestar lingüísticamente una idea o un sentimiento, pero parece que la forma más intensa de expresar algo es, paradójicamente, no expresarlo. 
